\title{
Paring Manuka Honey 10+ with Tumeric Powder and IV Antibiotic May Treat Complicated Infective Endocarditis Naturally
}

\author{
Dr. Hoang Nguyen, Md, Phd ${ }^{1 *}$, Dr. Quan Hong Nguyen, Md $^{2}$, Helen Thanh Nhi Dang ${ }^{3}$ \\ ${ }^{I}$ Department of Preventative Medicine, Charles R. Drew University (CDU)/UCLA School of Medicine and \\ Health Science, College Of Medicine, Los Angeles, California, USA \\ ${ }^{2}$ Department of Cardiology, Pham Ngoc Thach University of Medicine, HCM, Vietnam \\ ${ }^{3}$ Department of Biological Sciences, University Of California at Davis, California, USA
}

\begin{abstract}
*Corresponding Author: Dr. Hoang Nguyen, Department of Preventative Medicine, Charles R. Drew University (CDU)/UCLA School Of Medicine And Health Science, College Of Medicine, Los Angeles, California, USA, Email: hoangucla@yahoo.com
\end{abstract}

\begin{abstract}
Background: Mānuka honey 10+ is a honey produced from the nectar of the mānuka tree, Leptospermum scoparium. It is collected by honeybees foraging on its tree, which grows in New Zealand and Australia. This honey is commonly sold as a supplement, or alternative medicine. While several components in mānuka honey are studied for their potential bactericidal properties vivo. Superantigens toxins produced by Staphylococcal aureus have caused the heart valves functioning and kills thousands American every year on bacterial complication Infective Endocarditis (IE). This endotoxin is causing extensive activation of inflammatory cells; patients may present nonspecifically with fever, weight loss but not limited to other immunological reactions and hemorrhagic reaction. Complications in the form of systemic emboli from vegetation fragments can damage blood vessels within various organs, including brain, eye, spleen, kidney, pulmonary, and heart. Honey has broad spectrum antimicrobial bacteria activity specifically for staph bacteria due to its high osmolarity and high concentration of hydrogen peroxide. There is a synergistic potential action in treating wounds by combine both oxacillin and manuka honey against MRSA (methicillinresistant Staphylococcus aureus). Curcumin, the major constituent of Curcuma longa L. (Zingiberaceae family) is commonly called "turmeric", has used widely for cooking in Asian cuisine. At relatively nontoxic dose, it is known to be effective against Staphylococcus aureus (S. aureus). In vivo, turmeric powder will exhibit more potency effect when it used in combination with other IV antibiotic. Interestingly, paring of IV antibiotic regimen, manuka honey 10+ combine with turmeric powder might kill bacterial Staph Auerus Complicated Infective Endocarditis (IE) infection naturally.
\end{abstract}

Case Presentation: We report two cases of complicated infective endocarditis (IE), were cured by using manuka honey 10+, and turmeric powder in addition to IV antibiotic; two of the cases were associated with a septic emboli, and one case was associated with a renal infarction, immunology reactions (Janeway lesions and Osler Node), and a Hemorrhagic Reaction (Roth spots). One case of a patient with mitral valve endocarditis was complicated by intracerebral septic emboli, non-tender erythematous lesions on palms and soles, and Osler node tender on the tip of the finger, pulmonary hypoxia. A second case also had a splenic infarction, mycotic aneurysm and abscess associated with infected cardiac thrombi.

Conclusions: Infection endocarditis (IE) caused by Staphyloccus aures is more often frequently associated with complications. Complications can be associated with IE included: cardiac, neurologic, renal, musculoskeletal, but not limited to systemic complications such as metastatic infection, mycotic aneurysm, and embolization. Due to mortality rate increases when complications developed. Thus, aggressive empiric IV antibiotic combined with Manuka Honey 10+, and Tumeric Powder, would be the best regimen to cure this deathly infection disease.

Keywords: Infective Endocarditis, Manuka Honey, Tumeric Powder.

\section{BACKGROUND}

Manuka honey $10+$ has several antibacterial components, unlike other antibiotics; it does not induce resistance in bacteria. Its mechanism is altering the levels of protein synthesis components specifically at ribosomal protein 
location (1) (2). Bacteria's ability to reproduced and take over, were totally failed by using Manuka honey 10+; this experiment has done in the laboratory tests very successfully (3) (4). In the past 60 years, Tumeric has shown potent antibacterial activity and other pharmacological actions (5). Currently, Curcumin has been developed markedly into an antibiotic against $\mathrm{S}$. auerus and other bacteria strains. Globally, turmeric is marketed as a health supplement mainly for its antioxidant and anti-inflammatory properties. We report two cases of patients with complicated IE (Infective endocarditis) included neurological, renal, musculoskeletal, and systemic complications related to mycotic aneurysm, embolization, and metastatic infection; both cases were cured by paring Manuka Honey, Tumeric Powder with IV Antibiotic, would create successful wound healing.

\section{Case Presentation}

\subsection{Patient 1}

A 50-year-old man chief engineer worked for Boeing co. comes to the physician with malaise, anorexia, and fatigue for the past 6 months. He underwent an uncomplicated dental root canal procedure 7 months ago. His temperature is 36.0 C (97 F), blood pressure is $135 / 76 \mathrm{~mm} \mathrm{Hg}$, pulse is $90 / \mathrm{min}$, and respiration are $18 / \mathrm{min}$. An early diastolic murmur is heard at the left sternal border. CT chest, abdomen, pelvis with contrast was ordered; 1.5 Tesla magnet MRI brain with and without contrast also was ordered. CT scan result came back with no enhance masses are identified. There is no hydronephrosis, but there is a focal cortical scarring along lateral aspect of mild to lower left kidney. Suggestion from diagnostic radiologist there is a renal infarction or possible pylonephritis. MRI brain came result came back with several tiny foci of enhancement are identified, worrisome of embolic disease from unknown cause. A $2 \mathrm{cmm}$ focus of enhancement is detected in the left frontal white matter. A $3 \mathrm{~mm}$ focus of enhancement is detected in the right frontal sulci. A $2 \mathrm{mmm}$ focus of enhancement is detected in the posterior left cerebellum. A $5 \mathrm{~mm}$ focus of enhancement is detected the right posterior cerebellum. These lesions are associated with minimal edema, possibly due to their small size. A more subtle focus of peripheral enhancement in the inferior right cerebellum measures $3 \mathrm{~mm}$ in size and is associated with susceptibility. This could reflect a cavernous angioma. There is a trace mucosal thickening in the paranasal sinuses. Several tiny foci of enhancement are detected in the bilateral brain (Figure 1).

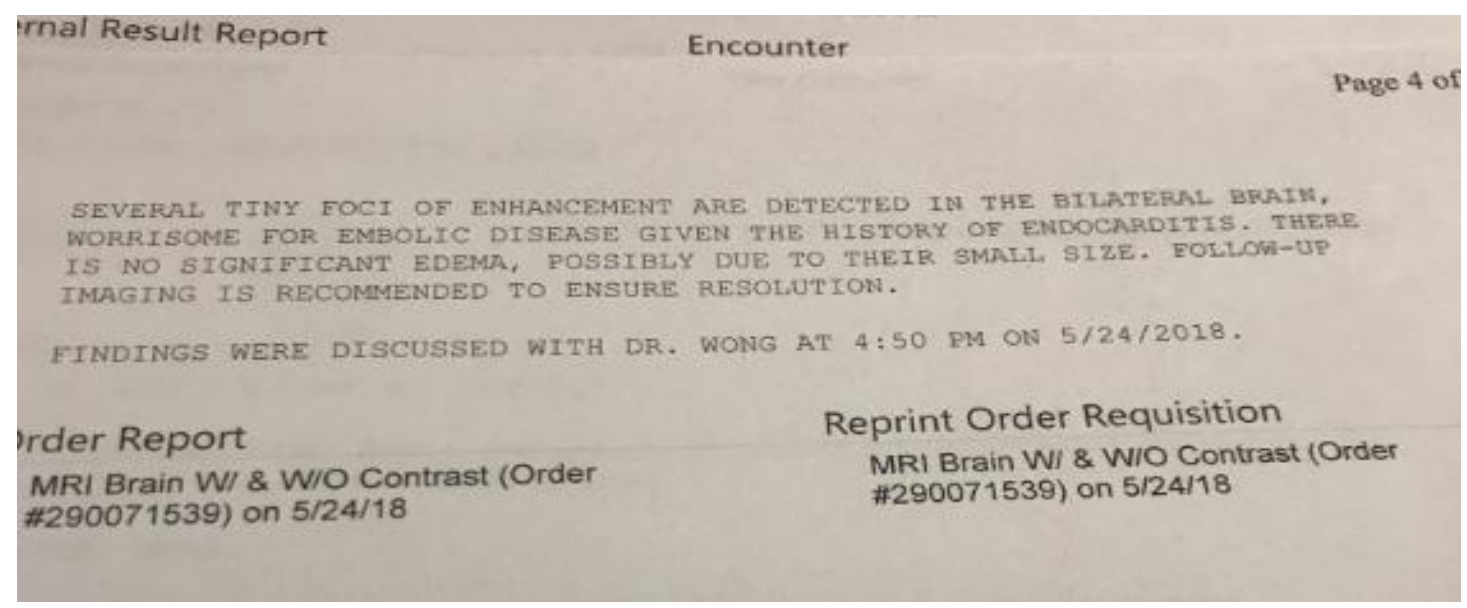

Figure 1

While waiting for TEE and serial of Blood culture result, Transthoracic Doppler Echocardiogram (TTE) result back with Ejaction Fraction (EF) is 60\%, Echogenic mass on the anterior leaflet of mitral leaflet $(94 \times 76 \mathrm{~mm})$ (Figure 2).

Echogenic mass on the anterior leaflet of mitral valve suspicious for vegetation. Transthoracic echocardiography (TTE) showed abnormal valve motion with evidence of vegetation but the patient's mean diastolic pressure gradient was elevated at $10 \mathrm{mmHg}$. On TEE, there is evidence of mitral valve vegetation with ecogenic mass up to $2.3 \mathrm{~cm} \times 2.2 \mathrm{~cm}$ on the anterior leaflet. CT surgeon and cardiologist decided to have open heart surgery to remove the vegetation ASAP. However, patient and his family member refused surgery plan, and 
decided to treat empiric with Ceftriaxone $2 \mathrm{mg}$ IV q24 and Daptomycin $8 \mathrm{mg} / \mathrm{kg}$ IV q24 for 42 days through the PICC line. After completed 6 weeks of IV antibiotic through the PICC, cardiologist decided to reassess patient again by ordered TEE (Figure 2).

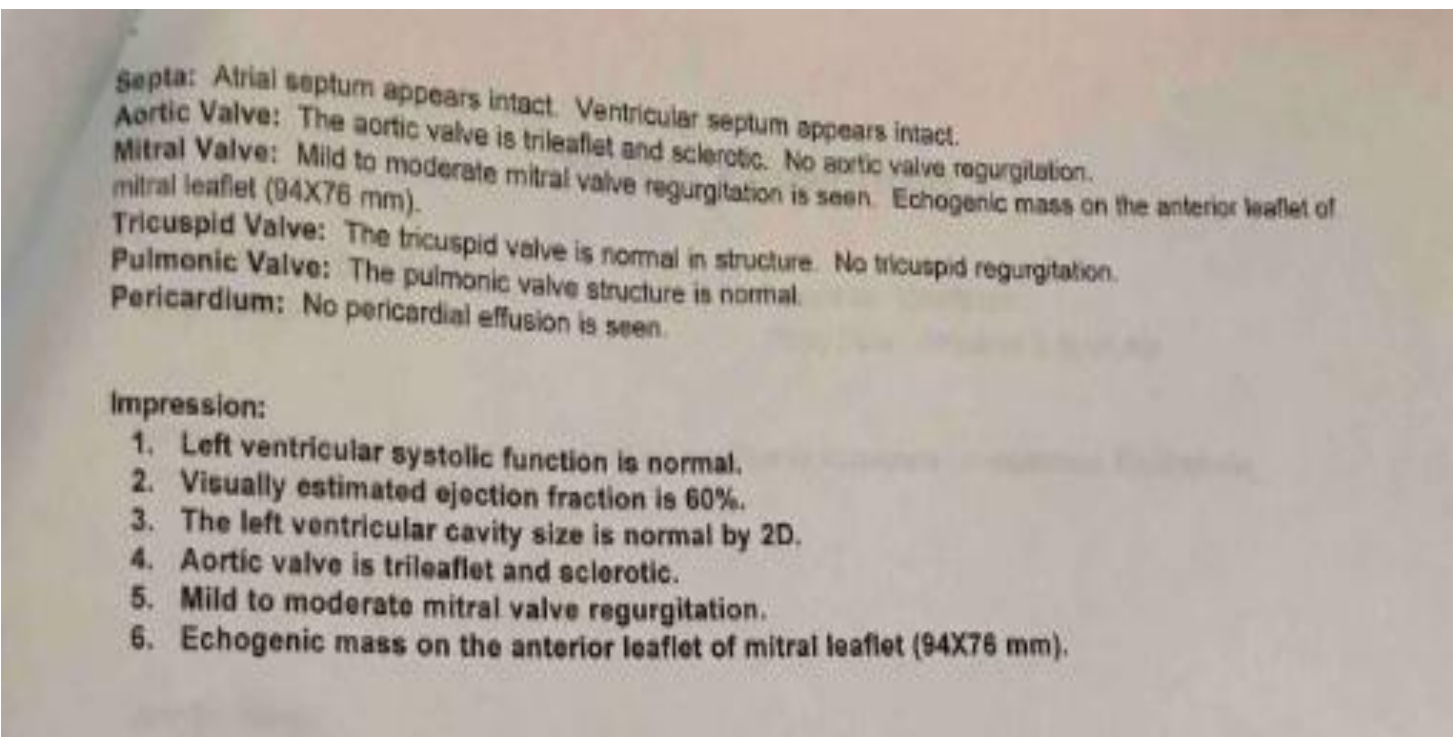

Figure 2

There is evidence of mitral valve vegetation with ecogenic mass up to $2.4 \mathrm{~cm} 2.1 \mathrm{~cm}$ on the anterior leaflet. CT surgeon and cardiologist still insisted to have open heart surgery ASAP to prevent cardio-neurovascular events. Instead, patient decided to take two teaspoonful of Turmeric powder, two teaspoonful of Manuka
Honey $10+$ were bought from Trader Joe's supermarket store, and taken as three times a day, and extend with Ceftriaxone 2mg IV q24, Daptomycin $8 \mathrm{mg} / \mathrm{kg}$ IV q24 to two more weeks and re-assess again with TEE on week \#8. TEE results come back with vegetation of $0.5 \mathrm{~cm}$, and result of culture is negative (Figure 3 ).

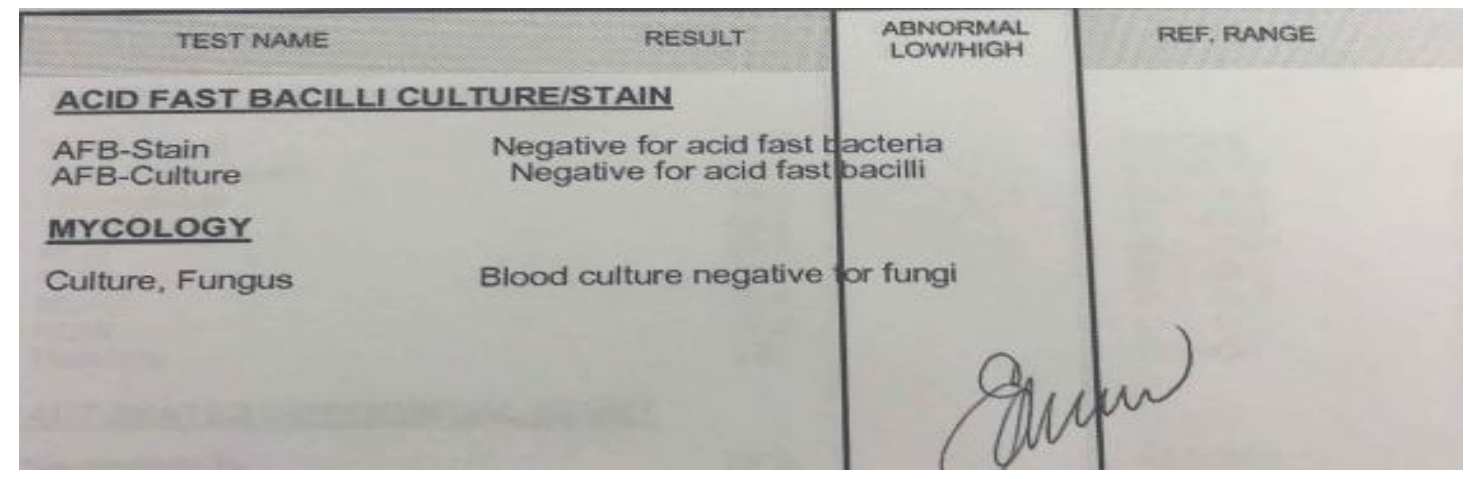

Figure 3

Figure3: It showed negative result after taken paring Manuka Honey 10+, Tumeric Powder and IV antibiotic regimen.

\subsection{Patient 2}

65-year-old Vietnamese women presented with generalized weakness and localized headache. Her medical h/o included DM type 2 and hypertension. Her physical examination revealed her blood pressure was $162 / 83 \mathrm{mmHg}$ and her pulse rate was 90 beats/minute. The patient developed a fever $\left(39.2{ }^{\circ} \mathrm{C}\right)$ after 4 weeks, without a definite source of infection. CMP lab test result came back with high creatinine level $2.0 \mathrm{mg} / \mathrm{dl}$, acute kidney injury has been diagnosed. Blood cultures revealed Staphylococcus aureus sensitive to Vancomycin. TEE revealed a globular, mobile, echogenic mass $(2.3 \times 1.0 \mathrm{~cm})$ (Figure 2). A peripherally inserted central catheter (PICC) was placed; consultant infection disease physician has ordered Ceftriaxone 2mg IV q24 and Daptomycin $8 \mathrm{mg} / \mathrm{kg}$ IV q24 for 42 days through the PICC line. After 2 weeks of treatment, her fever was subsided, but a vegetation and persistent septicemia were still the concern for 
all the team. We performed coronary angiography prior to possible valve surgery and observed no significant coronary obstruction. CT surgeon and cardiologist recommended valve surgery but the patient's husband refused. Patient's husband refused all surgery because of the high morbidity and mortality risk. Patient's husband decided to take Manuka Honey 10+, Tumeric powder in addition to current IV antibiotic regimen. After 3 weeks of using additional alternative medicine, TEE was ordered again; surprisingly, there is a nonmobile, echogenic mass $(0.4 \times 0.3 \mathrm{~cm})$, very insignificantly size, were attached to the tricuspid valve. Patient appeared as non-febrile, vital sign was normal; patient was discharged and followed up bi-monthly visit.

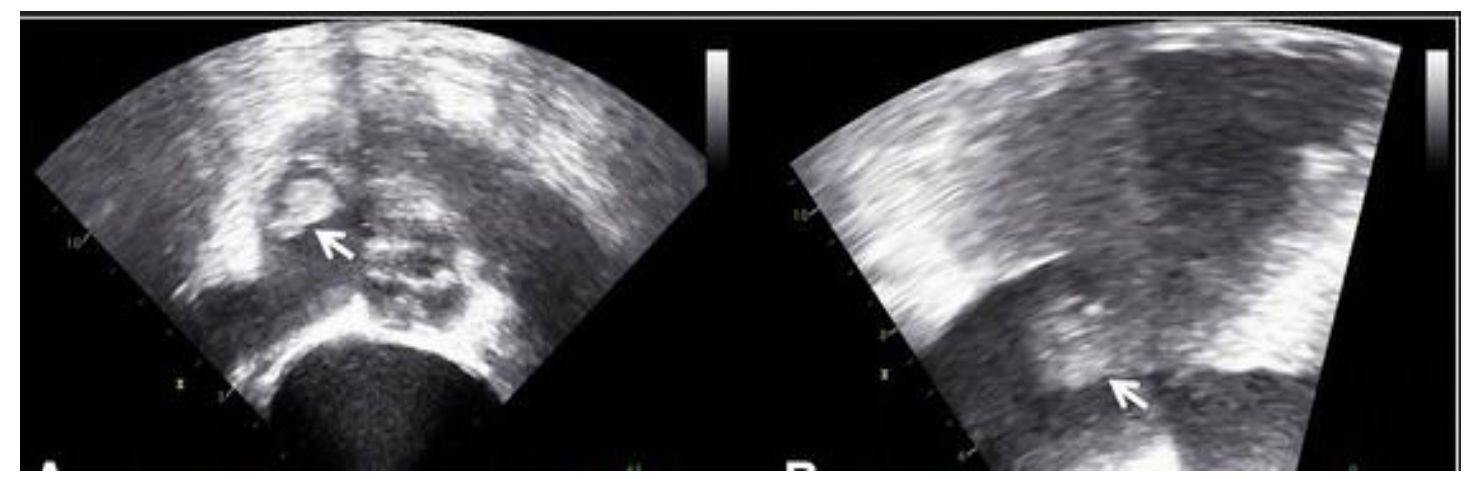

Figure 4

Fig 4(L) and 4(R) of Transesophageal Echocardiography revealing a globular, mobile, echogenic mass $(2.3 \times 1.0 \mathrm{~cm}$; arrows $)$ attached to the tricuspid valve

\section{DISCUSSION}

Manuka honey is sourced from nectar collected by honeybees foraging on the manuka tree, which grows in New Zealand and Australia (1). Many different types of honey have been used in medical remedies for thousands of years, both for antiseptic purposes and for the enhancement of chronic wound healing. Manuka honey has a very high level of dihydroxyacetone which produces methylglyoxal. Methylglyoxal is noted to not only have antibacterial properties, but also be bactericidal. This information was extracted from research that studied the effects of manuka honey on three different types of bacteria: Streptococcus pyogenes, Pseudomonas and Staphylococcus aureus (MRSA-15). The researchers were able to conclude that manuka honey does indeed affect the structure and activity of these three different types of bacteria. Adding manuka honey to the IV antibiotic therapy seemed to have potentiated the efficacy of the drug. The minimum inhibitory concentration (MIC) or minimum bactericidal concentrations, was used to measure this. Either test will measure the concentration of the drug that is needed to either kill the bacteria or retard its growth. For this reason manuka honey can be found in many patented wound care products, as it is regarded as a very significant component of topical ointments for superficial skin infections.
In addition to Staphylococcus aureus's susceptibility to manuka honey, it is also vulnerable to Curcumin. Curcumin, like manuka honey, also exhibits potent activity against a highly resistant strain of Staphylococcus aureus, MRSA, both alone and when combined with antibiotics. This is a very effective dynamic feature of many bactericidal products and curcumin seems to potentiate it. Pairing manuka honey 10+, turmeric powder and current IV antibiotic, this concoction will create a very strong antibiotic effect that may be capable of fighting off the complication of IE (Infective endocarditis). Similar to other natural remedies, this recipe of Manuka Honey $10+$, and Tumeric powder don't have any adverse effects on those using it, and can even be taken as the supplemental form.

\section{Conclusions}

Infectious endocarditis can bring about a host of other systemic complications, including renal, neurologic and musculoskeletal. Complicated infectious endocarditis significantly increases the mortality rate. For that reason, healthcare professionals must be astute and aggressive in rendering therapy. Treatment must include 
intravenous antibiotic therapy, and not withholding renown alternative methods such as Manuka Honey 10+ and/or Turmeric. There is sufficient research and evidence to unequivocally state Manuka Honey +10 and Turmeric are effective antimicrobial and antiinflammatory agents, respectively. Because of the defensing-1 protein, Manuka Honey +10 may even combat antibiotic resistant microorganisms. Therefore, adding these two natural elements may increase the efficacy of standard medical therapy of said conditions, thereby giving modern day medicine a superior edge.

Because the mortality rate increases with complications, aggressive antibiotic therapy combined with alternative medicine such as Manuka Honey 10+, Tumeric Powder will speed up the healing process and decrease inflammation reaction phase. Honey contains a protein known as defensing-1 which is produced by the bees. The protein is important in fighting against antibiotic-resistant microorganisms. When the two ingredients are combined with antibiotic, they form a highly potent and fastacting antibiotic, which leads to healing.

\section{REFERENCES}

[1] Carter, D. A., Blair, S. E., Cokcetin, N. N., Bouzo, D., Brooks, P., Schothauer, R., \& Harry, E. J. (2016). Therapeutic Manuka Honey: No Longer So Alternative. Frontiers in Microbiology, 7, 569. http://doi.org/ 10.3389/fmicb.2016.00569

[2] Jenkins R., Cooper R. (2012). Improving antibiotic activity against wound pathogens with manuka honey in vitro. PLoS ONE 7:e45600 10.1371/journal.pone.0045600

[3] Müller P., Alber D. G., Turnbull L., Schlothauer R. C., Carter D. A., Whitchurch C. B., et al. (2013). Synergism between Medihoney and rifampicin against methicillinresistant Staphylococcusaureus (MRSA). PLos ONE 8:e57679 10.1371/journal.pone.0057679

[4] Liu M., Lu J., Müller P., Turnbull L., Burke C. M., Schlothauer R. C., et al. (2014). Antibioticspecific differences in the response of Staphylococcus aureus to treatment with antimicrobials combined with manuka honey. Front. Microbiol. 5:77910.3389/ fmicb.2014.00779

[5] Schraufstätter E., Bernt H. Antibacterial action of curcumin and related compounds. Nature. 1949;164(4167):456-457. doi: 10.1038/ 164456a0. [PubMed] [CrossRef]

Citation: Hoang Nguyen, Quan Hong Nguyen, Helen Thanh Nhi Dang. Paring Manuka Honey 10+ with Tumeric Powder and IV Antibiotic May Treat Complicated Infective Endocarditis Naturally. ARC Journal of Cardiology. 2019; 5(1): 11-15. doi:dx.doi.org/ 10.20431/2455-5991.0501003.

Copyright: (1) 2019 Authors. This is an open-access article distributed under the terms of the Creative Commons Attribution License, which permits unrestricted use, distribution, and reproduction in any medium, provided the original author and source are credited. 